International Journal of Research in Advent Technology, Vol.7, No.5, May 2019

E-ISSN: 2321-9637

Available online at www.ijrat.org

\title{
A Novel Approach on Convolutional Neural Network for Face-Based Age and Gender Recognition
}

\author{
P V Narsimha Raju, K Bhargava Kiran \\ PG Scholar, Dept. of CSE, Vishnu Institute of Engineering Technology, Bhimavaram. \\ Assistant Prof, Dept. of CSE, Vishnu Institute of Engineering Technology, Bhimavaram.
}

\begin{abstract}
Age and gender order has turned out to be pertinent to a broadening measure of uses, especially coming about to the rising of social stages and online networking. In any case, execution of existing systems on genuine images is still on a very basic level missing, particularly when considered the enormous bounced in execution beginning late detailed for the related assignment of face affirmation. In this paper we demonstrate that by learning depictions utilizing significant Convolutional Neural Systems (CNN), an immense development in execution can be acquired on these errands. To this end, we propose a direct Convolutional Neural System outlining can be used despite when the measure of learning data is confined. We review our methodology on the current audience benchmark for age and gender classification and show it to fundamentally outmaneuver current state-of-the-art techniques.
\end{abstract}

Keywords: Age Estimation; Classification; Gender Recognition; Human Face; Neural Network.

\section{INTRODUCTION}

Age and gender assume essential parts in social between exercises. Lingos hold particular welcome and sentence structure rules for men or ladies, and as often as possible differing vocabularies are used while keeping an eye on senior residents contrasted with adolescents. Notwithstanding the fundamental parts these attributes play in our regular day to day existences, the ability to subsequently evaluate them decisively and constantly from confront image is as yet far from tending to the necessities of business applications. This is particularly astounding while at the same time considering late claims to super-human limits in the related errand of face acknowledgment. Past approaches to manage evaluating or requesting these properties from confront images have relied upon contrasts in facial component measurements or "modified" face descriptors. Most have used portrayal designs formed particularly for age or gender introduction estimation endeavors and others. Maybe a couple of these past methodologies were proposed to deal with the various troubles of unconstrained imaging conditions. Also, the machine learning systems used by these structures did not totally manhandle the tremendous amounts of image cases and data open through the Internet remembering the true objective to improve portrayal limits.

In this paper we try to close the gap between programmed confront acknowledgment capacities and those of age and gender orientation characterization procedures. To this end, we take after the productive example set around late face acknowledgment structures: Face acknowledgment frameworks depicted in the latest couple of years have exhibited that colossal progression can be made by the use of significant convolutional neural systems $(\mathrm{CNN})$. We demonstrate near increments with fundamental framework building, created by considering the fairly obliged availability of exact age and gender orientation arrangement names in existing face data sets.

\section{RELATED WORK}

Before delineating the proposed methodology we brief survey related frameworks for age and gender classification and give a diagram of critical convolutional systems.

Age Classification: The issue of therefore removing age related attributes from facial images has growing thought starting late and various procedures have been advanced. A point by point diagram of such techniques can be found and all the more starting late. We observe that paying little mind to our consideration here on age gather portrayal instead of correct age estimation (i.e., age relapse); the investigation joins procedures expected for either attempted. Early methods for age estimation rely upon finding out extents between different estimations of facial highlights. At the point when facial highlights (e.g. eyes, nose, mouth, jaw, et cetera.) are kept, their sizes and partitions are estimated, extents between them are determined and used for orchestrating the face into different age characterizations as showed by hand-made standards. All the more starting late, usages a relative method to manage display age development in subjects less than 18 years old. As those systems require exact 
International Journal of Research in Advent Technology, Vol.7, No.5, May 2019

\author{
E-ISSN: 2321-9637 \\ Available online at www.ijrat.org
}

limitation of facial components, testing issues are autonomous from any other individual, they are inadmissible for in-the-wild images which one may like to find on social platform.

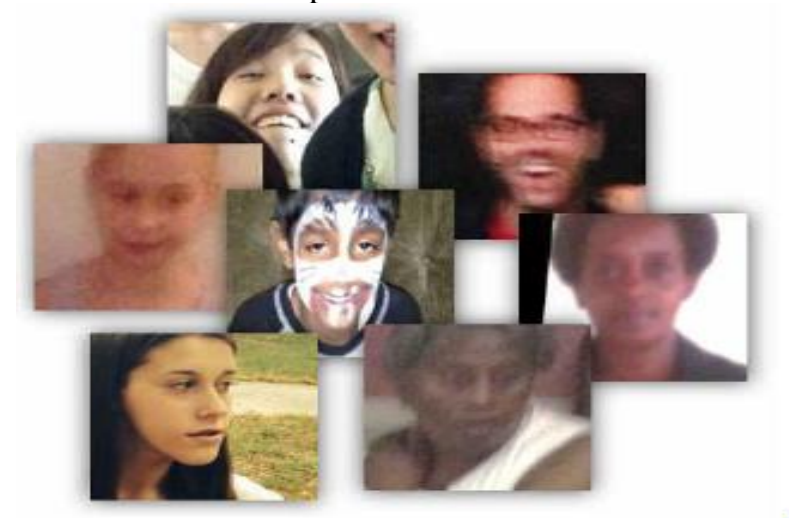

Fig. 1 Faces from the Audience benchmark for age and gender classification.

On a substitute calling are systems that address the creating method as a subspace or a complex. An obstruction of those frameworks is that they require data about the image to be close frontal and all that much adjusted. These frameworks in like way introduce test works out as expected just on compelled informational indexes of close frontal images. Once more, in like manner, such procedures are ill-suited for unconstrained images. Not precisely the same as those portrayed above are techniques that use contiguous parts for relating to confront images. Gaussian Mixture Models (GMM) was utilized to the diffusing of facial patches. GMM were utilized again to address the disseminating of near to facial estimations, however viable descriptors were utilized rather than pixel patches. At last, rather than GMM, Hidden-Markov-Model, super-vectors was utilized as a bit of confront fix transports.

Gender Classification: A point by point investigation of gender orientation order course of action systems can be found and all the more starting late. Here we quickly survey noteworthy systems. One of the early strategies for gender orientation order portrayal used a neural system framework arranged on a little game plan of close frontal face images. The united 3D structure of the head (obtained using a laser scanner) and image forces were used for gathering gender order. SVM classifiers were used, associated particularly to image forces. Rather than using SVM, used AdaBoost for a similar reason, here afresh, associated with image powers. Finally, point of view invariant age and gender orientation grouping portrayal was displayed. All the more starting lateused the Webers Local synthesis Descriptor for gender orientation grouping affirmation, displaying close perfect execution on the FERET benchmark. Power, shape and surface components were used with shared information, again drawing near perfect outcomes on the FERET benchmark.

Deep Convolutional Neural Networks One of the essential usages of convolutional neural systems (CNN) is perhaps the LeNet-5 framework delineated for optical character affirmation. Stood out from current significant $\mathrm{CNN}$, their framework was for the most part humble due to the confined computational resources of the time and the algorithmic challenges of getting ready more noteworthy frameworks. Despite the way that much potential laid in more significant CNN outlines (frameworks with more neuron layers), similarly starting late have they got the opportunity to be overwhelming, after the passionate addition in both the computational power, the measure of getting ready data immediately available on the Internet, and the change of more feasible systems for planning such complex models. One later and exceptional case is the use of significant $\mathrm{CNN}$ for image grouping in view of the testing Image net benchmark.

\section{A CNN FOR AGE AND GENDER ESTIMATION}

Gathering a substantial, markedimage getting ready set for age and gender orientation estimation from informal community image documents requires either access to singular information regarding the matters appearing in the images, which is frequently private, or is repetitive to physically name. Data sets for age and gender estimation from genuine interpersonal organization images are thusly respectably obliged in measure and in a matter of seconds no match in measure with the considerably bigger image course of action data sets (e.g. the Image net dataset). Over fitting is typical issue, when machine learning develop procedures are used as a part of image aggregations. This issue is exacerbated while considering significant convolutional neural system frameworks in light of their tremendous amounts of model parameters.

System Architecture Our proposed framework configuration is used all through our tests for both age and gender orientation grouping request. It is portrayed in Fig. 2. The framework contains only three convolutional layers and two totally connected layers with minimal number of neurons. This, by relationship with the considerably greater models associated. Our choice of a framework diagram is prodded both from our yearning to reduce the risk of 
over fitting and what's more the method for the issues we are trying to disentangle: age gathering on the Audience set requires perceiving eight classes; gender orientation order needs only two classes. This stood out from, e.g., the ten thousand identity classes used to set up the framework used for confront affirmation.

Every one of the three shading channels is dealt with particularly by the framework. Images are at first rescaled to $256 \times 256$ and a result of $227 \times 227$ is supported to the framework. The three resulting convolutional layers are then described as takes after.

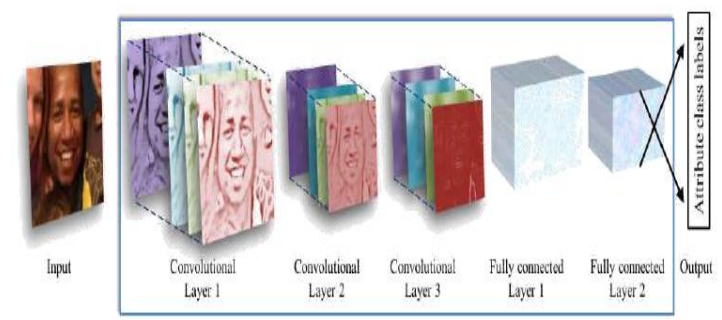

Fig. 2 Illustration of CNN architecture for age and gender classification

\section{Testing and Training Initialization:}

1. 96 channels of size $3 \times 7 \times 7$ pixels are associated with the data in the essential convolutional layer, trailed by an amended straight administrator (ReLU), a most extreme pooling layer taking the maximal estimation of $3 \times 3$ regions with two-pixel strides and a close-by response institutionalization layer.

2. The $96 \times 28 \times 28$ yield of the past layer is then dealt with by the second convolutional layer, containing 256 channels of size $96 \times 5 \times 5$ pixels. Again, this is trailed by ReLU, a greatest pooling layer and a nearby response institutionalization layer with indistinguishable hyper parameters from some time as of late.

3. At last, the third and continue going convolutional layer takes a shot at the $256 \times 14 \times 14$ blob by applying a course of action of 384 channels of size $256 \times 3 \times 3$ pixels, trailed by ReLU and a most extreme pooling layer.

The going with totally related layers is then described by:

4. A first totally related layer that gets the yield of the third convolutional layer and contains 512 neurons, trailed by a ReLU and a dropout layer.

5. A moment totally related layer that gets the 512dimensional yield of the fundamental totally related layer and again contains 512 neurons, trailed by a ReLU and a dropout layer.

6. A third, totally related layer which maps to the last classes for age or gender orientation order. Finally, the yield of the last totally related layer is urged to a fragile max layer that doles out probability for each class. The conjecture itself is made by carrying the class with the maximal probability for the given test image. The weights in all layers are instated with sporadic characteristics from a zero mean Gaussian with standard deviation of 0.01 . To extend this, we don't use pre-arranged models for instating the framework; the framework is readied, beginning with no outside help, without using any data outside of the images and the makes open by the benchmark. This, yet again, should be differentiated and CNN executions used for confront affirmation, where endless images are used for getting ready.

Network Training: Close to our use of slope framework outline, we apply two additional methodologies beyond what many would consider possible the peril of over fitting. To begin with we apply dropout learning (i.e. haphazardly setting the yield estimation of system neurons to zero). The framework joins two dropout layers with a dropout extent of 0.5 (half danger of setting a neuron's yield worth to zero). Second, we use data development by taking a subjective result of $227 \times 227$ pixels from the $256 \times 256$ image information and discretionarily reflect it in each forward-in reverse preparing pass.

Prediction: We attempted diverse things with two methods for using the framework as a part of demand to make age and gender orientation forecasts for novel faces:

- Center Crop: Feeding the framework with the face image, altered to $227 \times 227$ around the face center.

- Over-Sampling: We isolate five $227 \times 227$ pixel trim regions, four from the sides of the $256 \times 256$ face image, and an additional yield region from the point of convergence of the face. The framework is given each one of the five images, nearby their level reflections. Its last figure is taken to be the ordinary desire regard over each one of these assortments.

We have discovered that little misalignments in the Audienceimages, expedited by the various challenges of these images (obstructions, development cloud, et cetera.) can perceptibly influence the idea of our results. This second, finished testing procedure is expected to change for these misalignments, bypassing the prerequisite for improving course of action quality, yet rather particularly supporting the framework with various translated adjustments of a similar face.

\section{EXPERIMENT}

The Audience benchmark: We test the exactness of our CNN design using the starting late released Audience benchmark, expected for age and gender characterization. The Audienceimage set includes images subsequently exchanged to Flickr from PDA 


\section{International Journal of Research in Advent Technology, Vol.7, No.5, May 2019 \\ E-ISSN: 2321-9637 \\ Available online at www.ijrat.org}

contraptions. Since these images were exchanged without previous manual filtering, as is commonly the case on media site pages (e.g., images from the LFW gathering) or informal community destinations (the Group Image set), the conditions in these images are exceedingly unconstrained, mirroring countless present troubles of goes up against appearing in systems administration images. Audienceimages thusly find convincing assortments in head pose, lightning conditions quality, and anything is possible from that point.

The entire Audienceimage set social event fuses around $26 \mathrm{~K}$ images of 2,284 subjects. Table 1 records the breakdown of the aggregation into the unmistakable age arrangements. Testing for both age and gender is performed using a standard fiveoverlay, subjectparticular cross-endorsement tradition, described. We use the in-plane balanced adjustment of the faces, at first used. These images are used rather than additional a la mode course of action methods in order to feature the execution get credited to the framework configuration, instead of better pre-preparing.

TABLE I The Audience Faces Benchmark

\begin{tabular}{|c|c|c|c|c|c|c|c|c|c|}
\hline $\begin{array}{l}\text { Gender } \\
\text { Yenrs }\end{array}$ & 0.2 & 40 & 8.13 & $15: 20$ & 25.32 & 38443 & 48.53 & 60. & Total \\
\hline Jalle & 745 & 988 & 934 & 734 & 2308 & 1294 & $39 \%$ & 4.? & $819 \%$ \\
\hline Female & 682 & 1234 & 1360 & 919 & 2589 & 1056 & 433 & 427 & 94.1 \\
\hline Both & 1427 & 2162 & 204 & 1603 & 4897 & 2350 & 825 & 860 & 19587 \\
\hline
\end{tabular}

We test the time with same framework outline and used for all test folds of the benchmark and to be sure, for both gender orientation and age estimation assignments. This is performed with a particular true objective to ensure the authenticity of our results transversely finished folds, moreover to demonstrate the broad articulation of the framework design proposed here; a similar building performs well across finished different, related issues. We balance already announced outcomes with the results prepared by our framework. Our results join two methods for testing: focus edit and over-inspecting.

\section{CONCLUSION}

Disregarding the way that various past methods have watched out for the issues of age and gender gathering, starting in the not so distant past, a lot of this work has focused on obliged images taken in lab settings. Such settings don't adequately reflect appearance assortments typical to this present reality images in person to person communication destinations and online chronicles. Web images, how-ever, are not only all the more troublesome: they are moreover bounteous. The straightforward availability of gigantic image gatherings ace recordings propelled machine learning based systems with reasonably interminable getting ready data; however this data isn't by and large appropriately named for coordinated learning.

Taking outline from the related issue of face affirmation, we examine how well significant CNN perform on these assignments using Internet data. We give comes about a slope significant learning engineering intended to avoid over fitting as a result of the obstruction of compelled marked data. Our framework is "shallow" stood out from a bit of the late framework plans, thusly reducing the amount of its parameters and the shot for over fitting. We propel swell the degree of the planning data by dishonestly including trimmed variations of the images in our readiness set. The consequent system was attempted on the Audience benchmark of unfiltered images and appeared to on a very basic level beat late front line.

Two basic conclusions can be created utilizing our exploratory results. In any case, $\mathrm{CNN}$ can be used to give upgraded age and gender course of action comes about, despite considering the much minimal size of contemporary unconstrained image sets named for age and gender order. Second, the straight imposition of our model proposes that more included systems using all the all the more planning data may well have the capacity to do essentially upgrading outcomes past the one revealed here.

\section{REFERENCES}

[1]. F. Gao and H. Ai. "Face age classification on consumer images with gabor feature and fuzzy LDA method", In Advances in biometrics, pages 132-141. Springer, 2009.

[2]. X. Geng, Z.-H. Zhou, and K. Smith-Miles. "Automatic age estimation based on facial aging patterns", Trans. Pattern Anal. Mach. Intell., 29(12):2234-2240, 2007.

[3]. B. A. Golomb, D. T. Lawrence, and T. J. Sejnowski. Gendernet: "A neural network identifies gender from human faces", In Neural Inform. Process. Syst., pages 572-579, 1990.

[4]. S. E. Choi, Y. J. Lee, S. J. Lee, K. R. Park, and J. Kim. "Age estimation using a hierarchical classifier based on global and local facial features", Pattern Recognition, 44(6):1262-1281, 2011. 2

[5]. T. F. Cootes, G. J. Edwards, and C. J. Taylor. "Active appearance models", In European Conf. Comput. Vision, pages 484-498. Springer, 1998.

[6]. C. Cortes and V. Vapnik. "Support-vector networks", Machine learning, 20(3):273-297, 1995. 
[7]. E. Eidinger, R. Enbar, and T. Hassner. "Age and gender estimation of unfiltered faces", Trans. on Inform. Forensics and Security, 9(12), 2014.

[8]. Y. Fu, G. Guo, and T. S. Huang. "Age synthesis and estimation via faces: A survey", Trans. Pattern Anal. Mach. Intell., 32(11):1955-1976, 2010.

[9]. Y. Fu and T. S. Huang. "Human age estimation with regression on discriminative aging manifold", Int. Conf. Multimedia, 10(4):578$584,2008$.

[10]. K. Fukunaga. "Introduction to statistical pattern recognition", Academic press, 1991.

[11]. T. Ahonen, A. Hadid, and M. Pietikainen. "Face description with local binary patterns: Application to face recognition", Trans. Pattern Anal. Mach. Intell., 28(12):2037-2041, 2006.

[12]. S. Baluja and H. A. Rowley. "Boosting gender identification performance", Int. J. Comput. Vision, 71(1):111-119, 2007.

[13]. A. Bar-Hillel, T. Hertz, N. Shental, and D. Weinshall. "Learning distance functions using equivalence relations", In Int. Conf. Mach. Learning, volume 3, pages 11-18, 2003.

[14]. W.L. Chao, J.-Z. Liu, and J.-J. Ding. "Facial age estimation based on label-sensitive learning and age-oriented regression", Pattern Recognition, 46(3):628-641, 2013. 1, 2

[15]. K. Chatfield, K. Simonyan, A. Vedaldi, and A. Zisserman. "Return of the devil in the details: Developing deep into convolutional nets", arXiv preprint arXiv:1405.3531, 2014.

\section{About Authors: \\ P V NARSIMHA RAJU is currently pursuing his M.Tech (CSE) in,VISHNU INSTITUE OF TECHNOLGY, Bhimavaram, West Godavari, A.P.}

K BHARGAVA KIRAN is currently working as an Assistant Professor in Department of CSE, Vishnu Institute of Technology, Bhimavaram, West Godavari. His research includes networking and data mining. 